\title{
Neues aus dem Arzneimittelrecht
}

\author{
Walter Sibrowski Petra Krakowitzky \\ Institut für Transfusionsmedizin, Universitätsklinikum Münster, Deutschland
}

Transfusion MEDicine AND Hemotherapy veröffentlicht in dieser Ausgabe vier Aufsätze, die sich ausschließlich mit arzneimittelrechtlichen Problemen befassen, die durch Inkrafttreten der neuen Arzneimittel- und Wirkstoffverordnung (AMWHV) [1] wirksam geworden sind und in vielen Blutspendediensten zur Verunsicherung und manchmal auch zur Ratlosigkeit geführt haben. Die Autoren, kompetente Vertreter ihrer Arbeitsbereiche, haben sich dankenswerter Weise die Mühe gemacht, die wesentlichen Neuerungen im Arzneimittelrecht und die einzelnen rechtlichen Interpretationsrahmen auch für den praktisch tätigen Transfusionsmediziner in den wichtigen Kernfragen verständlich zu erläutern. Interessant ist, dass Horst Hasskarl [2] auch nach der 14. Arzneimittelgesetz(AMG)-Novelle [3] weiterhin ein Triumvirat der Verantwortlichkeiten sieht, das aus sachkundiger Person, Leiter der Herstellung und Leiter der Qualitätskontrolle besteht, während Wiebke Siegel [4] die Verantwortung ausschließlich bei der sachkundigen Person sieht.

Horst Hasskarl und Alice Ostertag [5] behandeln des Weiteren die Frage, welche Aufgaben im Bereich einer Blutspendeeinrichtung auf nichtärztliches Personal delegiert werden können. Die ausführliche Darstellung der haftungsrechtlichen Implikationen bei einem erweiterten Einsatz nichtärztlichen Personals in der Blutspende sowie der Ausblick darauf, dass die Einhaltung der $\S \S 3$ bis 12 Transfusionsgesetz (TFG) [6] in Zukunft Gegenstand auf Erteilung der Herstellungserlaubnis sein wird, sind hier nur zwei wichtige Aspekte dieser Arbeit. Gleichwohl wird ein Problem für die Transfusionsmedizin in Blutspendediensten und in den klinischen Bereichen nach der Lektüre offensichtlich: Das Transfusionswesen leidet unter einer dramatischen Überregulierung, die sich an der Anzahl und Art der gesetzlichen Bestimmungen und Verordnungen leicht verifizieren lässt. Und die Zukunftsaussichten versprechen keine Besserung. 2004 wurde die 12. AMG-Novelle verabschiedet. 2005 gab es die 13. und 14. Novelle des AMG sowie die erste Novelle des TFG und daraus resultierend eine Aktualisierung und Änderung der Hämotherapierichtlinien [7]. 2006 brachte die AMWHV. Und nun steht das Gewebege- setz [8] ins Haus, das wiederum zu grundlegenden Änderungen sowohl des AMG als auch des TFG führen wird.

Thomas Müller [9] gibt seinem Beitrag den Titel «Wieviel Arzneimittelrecht braucht die Transfusionsmedizin?» und gibt im Text auch die Antwort «Keep it simple». Man kann nur hoffen, dass sich diese Einsicht in allen Bereichen, die sich mit der Problematik «Blutversorgung» befassen, schnellstmöglich durchsetzt. Es ist längst an der Zeit, die in den letzten 15 Jahren angehäuften Gesetze und Verordnungen auf ihre Sinnhaftigkeit und Durchführbarkeit kritisch zu hinterfragen. Bei allem Regulierungszwang ist und bleibt Blut vom Menschen für therapeutische Zwecke ein «Unikat», das einmalig ist, wenn man sich die Mühe macht, es differenzierter zu betrachten, und nicht nur den Wirkstoff über Volumen, Gewicht, Zellzahl und Blutgruppe definiert. Jüngere Forschungsergebnisse machen evident, dass rote Blutzellen nicht nur eine Ansammlung von Hämoglobinmolekülen sind, die membranumhüllt 120 Tage im Blutkreislauf zirkulieren. Eine Betrachtung, die einseitig nur formalistische Regelungen aus dem Arzneimittelrecht kennt, kann diesem Sachverhalt jedenfalls nicht hinreichend gerecht werden. Man muss der Ausführung von Thomas Müller uneingeschränkt zustimmen, dass das Blut unter dem Regelungswerk des TFG besser untergebracht worden wäre. Die Rationale, die Blutspende dem AMG zuzuordnen, ist bei Kenntnis der physiologischen und pathophysiologischen Wirkungen und Nebenwirkungen des Bluts unverständlich bzw. rein willkürlich. Es ist an der Zeit, hier einen neuen Weg zu suchen und eine vernünftige Lösung zu finden, um die Versorgung der Patienten auch in Zukunft mit unverändert effizientem Sicherheitskonzept, das aber mit deutlich geringerem bürokratischem Überbau funktioniert, sicherzustellen. Der jetzt eingeschlagene Weg wird letztendlich bei Fortschreibung des bürokratischen Überbaus nicht mehr finanzierbar sein. Die Verschwendung von Ressourcen ist heute in vielen Bereichen der Gesellschaft ein Thema. Auch das Gesundheitswesen wird sich die Frage gefallen lassen müssen, inwieweit höhere Kosten für die Blutversorgung ohne eine nachvollziehbare Gegenleistung gerechtfertigt sind.

\begin{tabular}{ll}
\hline KARGER & @ 2007 S. Karger GmbH, Freiburg \\
$\begin{array}{l}\text { Fax +49 76145207 14 } \\
\begin{array}{l}\text { E-mail Information@Karger.de } \\
\text { www.karger.com }\end{array}\end{array}$ & $\begin{array}{l}\text { Accessible online at: } \\
\text { www.karger.com/tmh }\end{array}$
\end{tabular}

Prof. Dr. med. Dr. rer. nat. Walter Sibrowski

Institut für Transfusionsmedizin

Universitätsklinikum Münster

Domagkstraße 11, 48149 Münster, Germany

Tel. +49 251 83557-68, Fax -62

E-mail sibrowski@uni-muenster de 


\section{Literatur}

1 Verordnung über die Anwendung der guten Herstellungspraxis bei der Herstellung von Arzneimitteln und Wirkstoffen und über die Anwendung der guten fachlichen Praxis bei der Herstellung von Produkten menschlicher Herkunft (Arzneimittelund Wirkstoffherstellungsverordnung - AMWHV) vom 9. November 2006 (BGBL. I S. 2523).

2 Hasskarl H: Herstellungserlaubnisse im novellierten Arzneimittelrecht. Aufgaben und Verantwortung der sachkundigen Person nach § 14 AMG unter besonderer Berücksichtigung der Blutprodukte. Transfus Med Hemother 2007;34:DOI 10.1159/000100467.

3 Gesetz über den Verkehr mit Arzneimitteln in der Fassung der Bekanntmachung vom 12. Dezember 2005 (BGBL. I S. 3394), zuletzt geändert am 21. Dezember 2006 (BGBL. I S. 3367).
4 Siegel W: Die Umsetzung der 14. AMG-Novelle und der Arzneimittel- und Wirkstoffherstellungsverordnung in transfusionsmedizinischen Einrichtungen aus Sicht einer Landesbehörde. Transfus Med Hemother 2007:34: DOI 10.1159/000099164.

5 Hasskarl H, Ostertag A: Einsatz nichtärztlichen Personals im Rahmen der Blutspende. Transfus Med Hemother 2007;34:DOI 10.1159/000099162.

6 Gesetz zur Regelung des Transfusionswesens in der Fassung der Bekanntmachung vom 7.7.1998 (BGBL. I S. 1752), zuletzt geändert am 31.10.2006 (BGBL. I S. 2407).

7 Bekanntmachung der Richtlinien zur Gewinnung von Blut und Blutbestandteilen und zur Anwendung von Blutprodukten (Hämotherapie) gemäß $\S \S 12$ und 18 des Transfusionsgesetzes (TFG) (Novelle 2005). Bundesanzeiger, Jahrgang 57, Nr. 209a vom 5. November 2005
8 Gesetzesentwurf der Bundesregierung: Entwurf eines Gesetzes über Qualität und Sicherheit von menschlichen Geweben und Zellen (Gewebegesetz), www.bmg.bund.de/nn_603214/Shared Docs/ Gesetzestexte/Entwuerfe/Gewebegesetz,templateId = raw,property $=$ publicationFile.pdf/Gewebegesetz pdf.

9 Müller T: Arzneimittel- und Wirkstoffherstellungsverordnung (AMWHV): Wieviel Arzneimittelrech braucht die Transfusionsmedizin noch? Transfus Med Hemother 2007;34:DOI 10.1159/000200377. 